\title{
Imaging-Based Treatment Adaptation in Radiation Oncology
}

\author{
Esther G.C. Troost ${ }^{1,2}$, Daniela Thorwarth ${ }^{3}$, and Wim J.G. Oyen ${ }^{4,5}$ \\ ${ }^{I}$ MAASTRO Clinic, GROW School for Oncology and Developmental Biology, Maastricht University Medical Centre, Maastricht, The \\ Netherlands; ${ }^{2}$ Institute of Radiooncology, Helmholtz-Zentrum Dresden-Rossendorf, Dresden, Germany; ${ }^{3}$ Section for Biomedical \\ Physics, Department of Radiation Oncology, Eberhard Karls University, Tübingen, Germany; ${ }^{4}$ Department of Radiology and Nuclear \\ Medicine, Radboud University Medical Centre, Nijmegen, The Netherlands; and ${ }^{5}$ Institute of Cancer Research and Royal Marsden \\ NHS Foundation Trust, London, United Kingdom
}

\begin{abstract}
Learning Objectives: On successful completion of this activity, participants should be able to (1) understand the clinical implications of tumor characterization using different PET tracers before and during radiotherapy or chemoradiotherapy; (2) discuss the role of other functional imaging modalities (CT and MRI) in current oncology practice; and (3) introduce additional applications of PET imaging, such as in high-energy proton-beam therapy.
\end{abstract}

Financial Disclosure: Dr. Oyen receives research support and is on the advisory board and speakers' bureau for Bayer Healthcare. The authors of this article have indicated no other relevant relationships that could be perceived as a real or apparent conflict of interest.

CME Credit: SNMMI is accredited by the Accreditation Council for Continuing Medical Education (ACCME) to sponsor continuing education for physicians. SNMMI designates each JNM continuing education article for a maximum of 2.0 AMA PRA Category 1 Credits. Physicians should claim only credit commensurate with the extent of their participation in the activity. For CE credit, SAM, and other credit types, participants can access this activity through the SNMMI website (http://www.snmmilearningcenter.org) through December 2018.

In many tumor types, significant effort is being put into patienttailored adaptation of treatment to improve outcome and preferably reduce toxicity. These opportunities first arose with the introduction of modern irradiation techniques (e.g., intensity-modulated radiotherapy) combined with functional imaging for more precise delineation of target volume. On the basis of functional CT, MRI, and PET results, radiation target volumes are altered during the course of treatment, or subvolumes inside the primary tumor are defined to enhance the dosing strategy. Moreover, the probability of complications to normal tissues is predicted using anatomic or functional imaging, such as in the use of CT or PET to predict radiation pneumonitis. Besides focusing, monitoring, and adapting photon therapy for solid tumors, PET also has a role in verifying protonbeam therapy. This article discusses the current state and remaining challenges of imaging-based treatment adaptation in radiation oncology.

Key Words: PET/CT; functional CT; functional MRI; theranostics

J Nucl Med 2015; 56:1922-1929

DOI: 10.2967/jnumed.115.162529

$\mathbf{T}$ he arsenal of modern oncologic treatments includes surgery, high-precision radiotherapy, chemotherapy, targeted agents, immunotherapy, and combinations of these. Advanced imaging is a prerequisite for optimal patient management. In radiation oncology, PET and MRI play an increasing role in noninvasive characterization of the individual patient's tumor before and during radiotherapy to facilitate treatment adaptation. Persisting tumor activity or early

Received Jul. 6, 2015; revision accepted Sep. 22, 2015.

For correspondence or reprints contact: Esther G.C. Troost, Department of Radiation Oncology, University Hospital Carl Gustav Carus, Technische Universität Dresden, Fetscherstrasse 74, 01307 Dresden, Germany.

E-mail: esther.troost@uniklinikum-dresden.de

Published online Oct. 1, 2015.

COPYRIGHT (c) 2015 by the Society of Nuclear Medicine and Molecular Imaging, Inc. relapse after treatment places an unwanted burden on the patient from exposure to ineffective but still toxic treatment. A switch to more effective treatment is at best postponed but may be hampered or even prevented by previous treatment. Additionally, prolonged ineffective treatment is an increasing burden to the health care system in view of the high financial costs associated with modern therapies.

Rather than evaluating tumor response after administration of a substantial part or even completion of the treatment course, there is a shift toward early assessment and prediction of response and subsequent adaptation of treatment. Examples include radiation dose escalation or deescalation to the biologic subvolumes of voxels; addition, change, or omission of concurrent chemotherapy, enhancing the radiation effect but also increasing side effects; and tailored selection of targeted therapies, such as monoclonal antibodies and tyrosine kinase inhibitors.

Furthermore, PET provides valuable information on whether normal tissues surrounding the tumor can be spared with modern radiation treatment techniques, enables selection of patients benefiting from systemic therapy with radiolabeled agents to enhance the local dose, and may guide combined photon and proton irradiation techniques. Finally, advanced image analysis (radiomics) and combination of PET with functional anatomic imaging such as dynamic contrast-enhanced (DCE) CT, dual-energy CT, and diffusionweighted MRI (DWI) will irreversibly change assessment of tumors from mere measurement of size to multimodality delineation and characterization.

\section{FUNCTIONAL AND MOLECULAR IMAGING MODALITIES CURRENTLY AVAILABLE}

The most widely used PET radiopharmaceutical is ${ }^{18} \mathrm{~F}-\mathrm{FDG}$, which depicts tumor metabolism. Because numerous studies have shown the potential of ${ }^{18} \mathrm{~F}$-FDG PET for staging, delineating target volume, assessing response, and detecting relapse in a wide variety of cancers, along with the associated impact on management of patients, it has become the cornerstone of biologic imaging in oncology $(1,2)$. Although increased metabolism due to the 
Warburg effect is an important reflection of tumor aggressiveness, more specific tracers to depict distinct tumor characteristics have been developed. For several decades it has been known that one of the major biologic factors driving radiation resistance is tumor cell hypoxia. Hypoxia can be noninvasively visualized by PET imaging using dedicated tracers such as ${ }^{18} \mathrm{~F}$-fluoromisonidazole, ${ }^{18} \mathrm{~F}$-fluoroazomycin arabinoside, or ${ }^{18} \mathrm{~F}-\mathrm{HX} 4$ (3-7). Tumor cell proliferation can be depicted by the thymidine analog ${ }^{18} \mathrm{~F}$-fluorothymidine, which has been validated against histopathologic specimens in a broad variety of solid tumors and lymphomas (8-10). Apart from tracers depicting biometabolic tumor characteristics such as angiogenesis and apoptosis, radiotracers assessing tumor receptor expression, such as human epithelial growth factor receptor 2 and the epidermal growth factor receptor (EGFR), are increasingly being applied (11-14).

Besides novel radiopharmaceuticals, developments in hardware have revolutionized medical imaging. The introduction of combined PET/CT instead of stand-alone scanners has enhanced the potential and impact of PET and was responsible for the increasing application of molecular imaging in radiotherapy practice (15). More recently, integrated PET/MRI has further advanced the impact of multimodality imaging. With functional techniques for both CT (e.g., DCE CT and dual-energy CT) and MRI (e.g., DCE MRI and DWI) being in place, the simultaneous acquisition is likely to enhance adaptive treatment techniques.

\section{USE OF FUNCTIONAL MRI AND CT IN RADIOTHERAPY}

In view of its superior soft-tissue resolution, conventional MRI is of pivotal importance for detection, local staging, and delineation of many cancer types (e.g., pelvic, head and neck, and brain tumors and sarcoma). When MRI is incorporated into radiation treatment planning, dedicated image acquisition techniques may be necessary and other technical issues may arise. Especially, geometric distortions can be significant $(>10 \mathrm{~mm})$ and thus need to be identified and addressed in this era of high-precision radiotherapy. With MRI systems being integrated with linear accelerators (MR Linac), real-time imaging of tumor locations during the actual treatment delivery has been realized, facilitating precise delivery of dose to target while sparing surrounding normal tissues (16). It would seem straightforward to expand the use of MR Linac for continuous adaptation of radiotherapy, especially in tumors subjected to internal motion such as breathing.

In cervical cancer, it is well established that the rates of complete remission are favorable for MRI-guided brachytherapy as compared with conventional brachytherapy, resulting in high completeremission rates and a low frequency of local recurrence (17). As described by Kharofa et al. (18), the use of MRI during treatment in these patients will facilitate treatment adaptation, optimizing coverage of the target volume while minimizing dose to organs at risk. DWI holds great promise for adaptive radiotherapy, for it relies on restriction of the diffusion of water molecules in areas of high cellularity. This restriction of diffusion is expressed as the apparent diffusion coefficient (ADC). Although the use of MRI is already part of clinical practice in the diagnostic work-up of many tumor types, the role for DWI in adaptive radiotherapy is currently the subject of clinical studies. In an early study by Kim et al. (19), treatment-induced changes in ADC were predictive of response to chemoradiotherapy in patients with squamous cell carcinoma of the head and neck (HNSCC) as early as 1 wk after the start of therapy. In another study on HNSCC, it was shown that patients with lower tumor
ADCs after 4 wk of radiotherapy had a higher risk for locoregional recurrence (20). Schmid et al. (21) reported that MRI morphologic features predicted tumor response after chemoradiotherapy in 85 cervical cancer patients.

Besides static imaging, with or without contrast enhancement, MRI offers several noninvasive functional features. DCE MRI depicts perfusion and vascular permeability. By applying the appropriate pharmacokinetic models, DCE MRI provides semiquantitative data on fluid transfer between the bloodstream and the extravascular extracellular space. Blood oxygen level-dependent MRI provides information on the ratio between oxygenated and deoxygenated hemoglobin in the blood (22). Because deoxygenated hemoglobin is paramagnetic and oxygenated hemoglobin is not, blood oxygen level-dependent imaging can provide information about the hypoxic status of tumors. Furthermore, it is feasible to measure the oxygenation status of tumors and tissues with MRI-based techniques, using oxygen-dependent T1 relaxation times. An additional option is the use of MR spectroscopy to study metabolic changes in tumors, assessing the relative concentrations of cancer cell metabolites. In a promising French study, MR spectroscopy before radiotherapy allowed stratification of patients with glioblastoma multiforme according to therapy outcome (23). Combining all data provided by functional MRI into a multiparametric dataset provides an extensive characterization of tumors, such as has been shown in prostate cancer (24).

DCE CT is slowly finding its way into clinical studies assessing tumor perfusion and treatment response. In a pilot study on 15 rectal cancer patients treated with chemoradiotherapy, Sahani et al. (25) found tissue blood flow to decrease and mean transit time to increase after treatment. Baseline tissue blood flow and mean transit time separated responders from nonresponders. Similar results were reported for patients with colorectal cancer and hepatocellular carcinoma in a study monitoring the response to treatment, such as bevacizumab or sorafenib $(26,27)$, that blocks the vascular endothelial growth factor. Sauter et al. (28) extensively correlated DCE CT parameters (tissue blood flow, blood volume, volume transfer coefficient, and standardized perfusion value) with ${ }^{18} \mathrm{~F}$-fluorothymidine PET parameters $\left(\mathrm{SUV}_{\max }, \mathrm{SUV}_{\text {mean }}\right.$, and metabolic tumor volume) and immunohistochemical analysis (Ki-67, microvessel density) in 24 patients with non-small cell lung cancer (NSCLC). They reported that ${ }^{18}$ F-fluorothymidine PET measures correlated with Ki-67, whereas microvessel density correlated with blood flow and volume, as well as with volume transfer coefficient. Just recently, Tixier et al. (29) found a significant correlation between intratumoral heterogeneity on ${ }^{18} \mathrm{~F}$-FDG PET and tumor blood flow as detected by DCE CT in advanced-stage colorectal cancer. Dual-energy CT imaging - that is, simultaneous acquisition of 2 CT scans with different kilovoltages - will most certainly provide new possibilities for improving the accuracy of target volume delineation and tumor and normal-tissue characterization (Fig. 1).

\section{PET FOR RADIATION TREATMENT ADAPTATION AND NORMAL-TISSUE CHARACTERIZATION}

In recent years, several studies and metaanalyses have shown that modern molecular and functional imaging with PET, MRI, and functional MRI allows for imaging of prognostic parameters with respect to radiotherapy outcome $(4,30)$.

Some studies have recently investigated imaging strategies to assess the response to therapy at an early time point during radiotherapy or chemoradiotherapy. Van Elmpt et al. (31) assessed ${ }^{18}$ F-FDG PET 


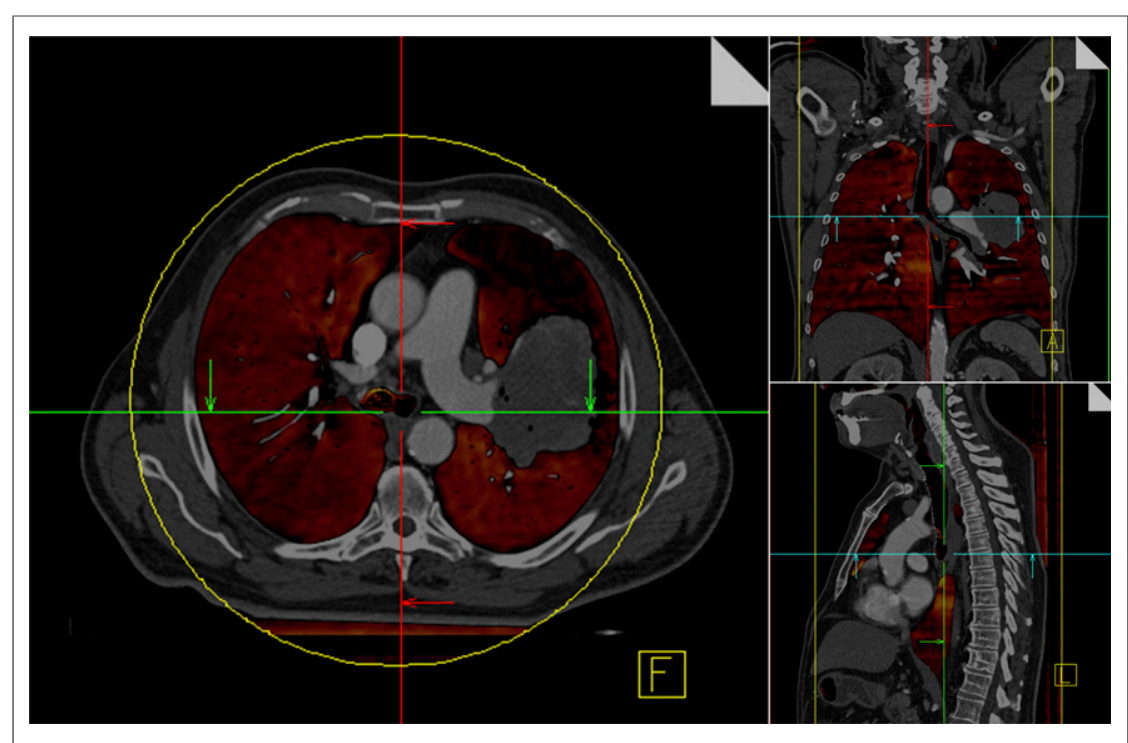

FIGURE 1. Perfused lung volume (color overlay) measured using contrast-enhanced dualenergy CT scan of patient with NSCLC tumor inside left lobe (shown in transverse, frontal, and sagittal views). Large perfusion defect surrounding tumor is clearly visible, most probably caused by obstruction of pulmonary vessels. (Courtesy of Wouter van Elmpt, Department of Radiation Oncology [MAASTRO Clinic], Maastricht University Medical Centre, Maastricht, The Netherlands, and Marco Das, Department of Radiology, Maastricht University Medical Centre, Maastricht, The Netherlands.)

uptake patterns before and 2 wk into therapy in 34 advanced-stage NSCLC patients and reported that a marked decrease in the ${ }^{18} \mathrm{~F}$ FDG PET signal between the two PET scans predicted higher overall survival. In a similar Dutch study, Usmanij et al. (32) showed that a decrease in total-lesion glycolysis of more than $38 \%$ during the first 2 wk of therapy was associated with a significantly longer progression-free survival.

Several recent studies have shown the prognostic value of hypoxia PET imaging with respect to radiotherapy outcome for a variety of solid tumors (33-36). A study published by Zips et al. (36) underlined the prognostic value of ${ }^{18} \mathrm{~F}$-fluoromisonidazole PET imaging before and after 2 wk of radiotherapy or chemoradiotherapy in 25 HNSCC patients, whereas baseline ${ }^{18} \mathrm{~F}$-fluoromisonidazole PET did not correlate with treatment outcome, providing a basis for the integration of hypoxia PET into radiotherapy planning for hypoxiadirected dose escalation strategies. A Danish study even found ${ }^{18} \mathrm{~F}$ fluoroazomycin arabinoside PET imaging to stratify patients into groups according to locoregional control (34). In a small study cohort, Dirix et al. (20) investigated different functional imaging modalities $\left({ }^{18} \mathrm{~F}-\mathrm{FDG}\right.$ and ${ }^{18} \mathrm{~F}$-fluoromisonidazole PET, T1- and T2weighted MRI, DWI, and DCE MRI) at various time points before and during primary radiotherapy in $15 \mathrm{HNSCC}$ patients. In addition to finding hypoxia PET to have prognostic value, the authors reported that tumors recurring locally or regionally during follow-up had significantly lower ADCs on DWI during and after radiotherapy and were significantly different on DCE MRI from lesions that remained controlled. Molecular imaging of prognostic parameters and biologic properties is an essential prerequisite for an individual adaptation of treatment.

Troost et al. (10), when repeatedly imaging 10 oropharyngeal cancer patients, found that an SUV decrease in the proliferation tracer ${ }^{18} \mathrm{~F}$ fluorothymidine preceded volumetric changes on CT. Subsequently, Hoeben et al. (37) assessed a larger cohort of 48 advanced-stage
HNSCC patients and reported a large decrease in the ${ }^{18} \mathrm{~F}$-fluorothymidine PET signal in the first 2 or 4 wk to be associated with a better 3-y disease-free survival (Fig. 2). Preliminary findings on ${ }^{18} \mathrm{~F}$-fluorothymidine PET in HNSCC patients receiving radiotherapy combined with the monoclonal antiEGFR antibody cetuximab need confirmation by a larger interventional study (38). ${ }^{18} \mathrm{~F}$ fluorothymidine may be a powerful tool to stratify patients and adapt treatment accordingly (e.g., additional antiproliferative treatment by means of chemotherapy or EGFR inhibition).

Because of the above findings from exploratory imaging studies, a few centers have started phase I or II studies to investigate the potential and limitations of individualized radiotherapy strategies adapted on the basis of functional imaging information acquired before or during radiotherapy $(31,39-41)$. To date, biologic adaptation strategies consist mainly of escalating the dose to functionally abnormal or radioresistant subvolumes inside the gross tumor volume (GTV), even though results from preclinical studies on this topic are still eagerly awaited. To systematically investigate the clinical feasibility and toxicity of dose escalation in HNSCC, Leclerc et al. (40) performed a phase I clinical trial in which therapeutic planning target volumes (PTVs) were treated using 3 consecutive dose levels of 69,72 , or 75 Gy in 30 fractions. The main results of the study were that dose escalation at this order of magnitude was effective and safe, with no differences in toxicity among the 3 treatment arms. The first dose-painting trial individually adapting the prescribed dose level on the basis of ${ }^{18} \mathrm{~F}-\mathrm{FDG}$ PET results was published in 2007 by Madani et al. (39). Within that phase I clinical trial, 41 HNSCC patients were treated in 2 arms with dose levels of 72.5 and 77.5 Gy to the ${ }^{18}$ F-FDG PET-derived biologic target volume using a simultaneous integrated boost technique. One treatment-related death was reported in the experimental arm, consequently halting the study. In 2011, the same Belgian group reported on another dose-painting study, escalating dose to a median of 80.9 or 85.9 Gy to the ${ }^{18}$ F-FDG PET-avid region inside the GTV using a dose-painting-by-numbers approach (42). For that study, 3 individually adapted treatment plans were generated: planning of fractions 1-10 and 11-20 was based on the ${ }^{18}$ F-FDG PET-derived biologic target volume, whereas fractions 21-32 were planned conventionally. In 6 of 21 patients recruited into that study, dose-limiting toxicity, that is, mucosal ulcers, was observed and thus the maximum tolerated dose was set at a median of 80.9 Gy. To date, the 2011 study of Madani et al. (42) has been the only one to report a maximum tolerated dose for dose escalation in HNSCC, suggesting that there is only a narrow window of additional dosing (total dose of approximately 70-80 Gy) available for personalized radiation strategies based on functional imaging. To reach additional efficacy in terms of locoregional control, other strategies of treatment intensification are warranted.

Two currently recruiting prospective phase II clinical studies-one on NSCLC (31) and the other on HNSCC, (43) — are investigating the potential for escalating radiation dose on the basis of ${ }^{18} \mathrm{~F}-\mathrm{FDG}$ PET. The PET-boost trial on advanced-stage NSCLC randomizes between dose escalation to the entire primary tumor volume and dose 


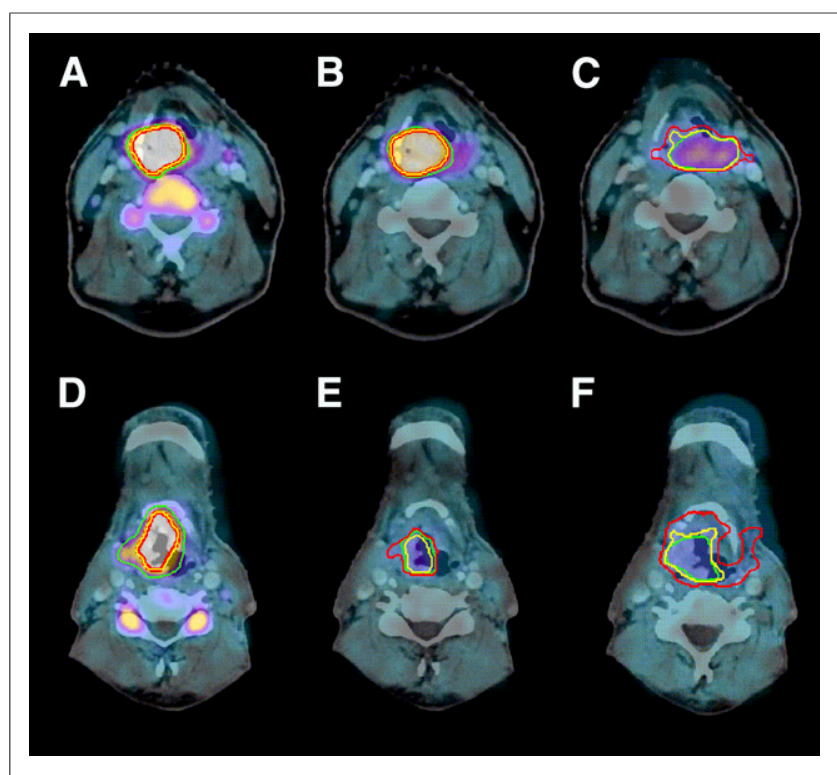

FIGURE 2. ${ }^{18}$ F-fluorothymidine PET/CT before therapy (A and D), in second week of therapy ( $B$ and $E$ ), and in fourth week ( $C$ and $F$ ). Top row shows slow decrease in ${ }^{18} \mathrm{~F}$-fluorothymidine uptake in patient with cT4N2bM0 supraglottic laryngeal carcinoma treated with chemoradiotherapy. This patient developed local recurrence $7 \mathrm{mo}$ after end of treatment and died of metastatic disease. Bottom row shows fast decrease of ${ }^{18} \mathrm{~F}$-fluorothymidine uptake in patient with cT3N1MO supraglottic laryngeal carcinoma treated with radiotherapy only. This patient developed no tumor-related event after 32 mo of follow-up. (Reprinted from (37).)

escalation to the ${ }^{18}$ F-FDG-avid region inside the GTV at the same integral dose while the dose to the affected lymph nodes remains unaltered (31). The ARTFORCE trial on HNSCC (43) has altered its design to randomize between standard irradiation (70 Gy in 35 fractions) and dose-painted redistribution of the radiotherapy based on $50 \%$ of the $\mathrm{SUV}_{\max }$ contour within the GTV (GTV ${ }^{18} \mathrm{~F}-\mathrm{FDG}$ PET), with a maximum total dose in the investigational arm of 84 Gy to $2 \%$ of the PTV GTV PET, which equals GTV ${ }^{18}$ F-FDG PET expanded by $3 \mathrm{~mm}$.

A German single-institution randomized phase II study is investigating the clinical feasibility of hypoxia dose escalation in HNSCC (41). Patients randomized into the experimental arm are treated with a $10 \%$ dose escalation, that is, $77 \mathrm{~Gy}$, to a hypoxic volume defined on the basis of dynamic ${ }^{18} \mathrm{~F}$-fluoromisonidazole PET data. The planned interim analysis for the first 20 patients recruited into this study showed that dose escalation on this order of magnitude is well tolerated (41). Furthermore, results from this study have confirmed a prognostic model relating individual tumor control probability to the dynamic ${ }^{18} \mathrm{~F}$-fluoromisonidazole PET data established in an earlier study (44).

To clinically implement personalized radiotherapy based on functional imaging data, several methodologic issues need to be considered. Thus far, radiotherapy planning and dose calculation are performed predominantly on the basis of a dedicated planning CT scan. As a consequence, some of the functional imaging data have to be registered with robust, accurate, previously validated algorithms (45). Hence, different algorithms and parameter settings may be required for the registration of data acquired from different anatomic regions using different imaging techniques. To minimize registration errors and guarantee a robust translation of the functional voxel information into radiotherapy planning information, it is recommended that functional PET or MR images be acquired with the patient in the radiotherapy position $(24,46)$. To account for biologic and anatomic changes during radiotherapy, adaptive treatment strategies also appear promising for biologically individualized radiotherapy based on functional PET or MRI (47).

Modern imaging equipment increasingly enables the acquisition of multiple functional images in parallel or sequentially at a close interval. Houweling et al. (48) found that ${ }^{18}$ F-FDG PET information differed from ADC maps derived from DWI data in 18 HNSCC patients, resulting in different targets for potential dose painting strategies. Another study proposed a statistical model to combine different functional MRI datasets to derive a voxel-based probability of tumor presence for more accurate automatic contouring (49). The proposed model was validated against pathologic information from the prostatectomy specimens of 87 prostate cancer patients (49). With the increasing availability of multiparametric functional imaging data from hybrid scanners such as PET/ MRI, methods to combine data containing different biologic information-for uses other than just target volume delineationappear promising. Consequently, Alber and Thorwarth (50) proposed integrating a voxel-based probability of tumor presence directly into the optimization process for radiotherapy planning. This formalism also takes into account the varying sensitivities and specificities of different imaging modalities, as well as other sources of uncertainty, such as patient movement. An extension of this methodology would also allow multiparametric image information to be combined to derive a probability map of radiation resistance inside the GTV. Figure 3 presents an example in which ${ }^{18}$ F-fluoromisonidazole PET data and ADCs derived from DWI were used to calculate a radiation sensitivity map that subsequently served as input for probability dose painting taking into account voxel-based radiation resistance. Such approaches require validated classification data for several different functional datasets, which so far have been available only from preclinical data.

A recently proposed approach to using imaging information for patient stratification and treatment personalization is radiomics. This methodology comprehensively quantifies tumor phenotypes by extracting a large number of quantitative image features $(51,52)$. In a recent extensive study, Aerts et al. (51) presented a radiomic analysis of 440 features quantifying tumor image intensity, shape, and texture, which were extracted from the CT data of more than 1,000 NSCLC and HNSCC patients. The authors concluded that a prognostic radiomics signature associated with underlying gene-expression patterns is able to capture intratumor heterogeneity and may thus identify a general prognostic phenotype in both patient cohorts. Consequently, this emerging field converting medical images into minable data is a potentially powerful tool for radiotherapy personalization.

Apart from tumor characterization and target volume definition, PET enables the detection of radiation-sensitive tissue subvolumes in nontumorous tissues, such as lung tissue prone to developing radiation pneumonitis. In a study by Petit et al. (53), a cohort of 101 prospectively recruited NSCLC patients was retrospectively analyzed to investigate the relationship between pretreatment ${ }^{18} \mathrm{~F}$ FDG uptake in the lungs, the delivered radiation dose, and radiationinduced lung toxicity (RILT). The study revealed that the risk of RILT increased with pretreatment ${ }^{18} \mathrm{~F}-\mathrm{FDG}$ uptake in the lungs. Consequently, the risk of RILT may decrease when lung subvolumes with high ${ }^{18}$ F-FDG uptake are avoided by applying dedicated 

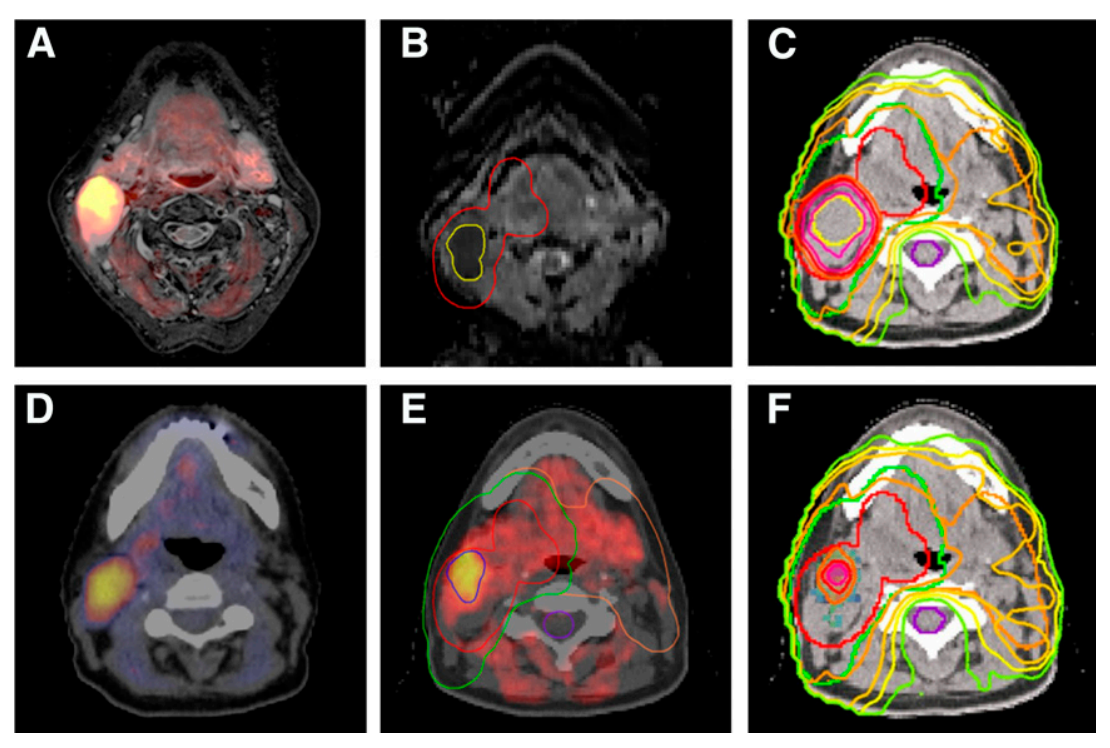

FIGURE 3. A 56-y-old woman with base-of-tongue HNSCC who underwent multiparametric functional PET/MRI before start of radiotherapy. (A) Combined ${ }^{18} \mathrm{~F}$-fluoromisonidazole PET/MR image $3 \mathrm{~h}$ after injection (T2-weighted turbo inversion recovery magnitude). (B) ${ }^{18} \mathrm{~F}$-fluoromisonidazole PET image registered to planning CT image showing different PTVs. (C) ADC map derived from DWI including PTV containing low ADCs inside PTV of first order. (D) Example radiotherapy plan with dose escalation of $20 \%$ prescribed to PTV containing low ADCs. (E) Probability map for radiation resistance of tumor derived from combination of ${ }^{18} \mathrm{~F}$-fluoromisonidazole PET and ADC. (F) Dose-painting radiotherapy plan directly optimized on probability map shown in E. Radiation treatment plans were optimized using treatment-planning system Hyperion for VMAT treatment with 2 arcs and 6-MV photons. PTVs of interest are 70 Gy (red), PTV containing low ADCs (yellow), fluoromisonidazole (blue), 60 Gy (green), 54 Gy (orange), and spinal cord (purple). Isodose lines (from lowest to highest) are $45,51,57.5,66.5,73,77$, and 80 Gy.

radiation planning techniques. A similar study investigated the potential of CT imaging to measure RILT (54). Here, changes in the Hounsfield units of CT scans acquired before and 3 mo after radiotherapy were correlated with RILT. The authors reported that changes in Hounsfield units correlated linearly with radiation dose, with the implication being that changes in Hounsfield units identify the whole range of individual radiosensitivity on a continuous, quantitative scale. Moreover, a study by Nijkamp et al. (55) found a positive correlation between radiation dose and severity of acute esophagitis as detected by ${ }^{18}$ F-FDG PET uptake after concurrent chemotherapy and radiotherapy in 82 NSCLC patients. Thus, functional imaging with ${ }^{18} \mathrm{~F}-\mathrm{FDG}$ PET can assess the radiation sensitivity of normal tissue to enable better future treatment.

\section{PET FOR IN VIVO TREATMENT VERIFICATION OF PROTON AND CARBON ION THERAPY}

Proton and carbon ion therapy are emerging techniques indicated for a variety of solid tumors (e.g., pediatric tumors, chondrosarcoma, chordoma, and neurooncologic tumors) to increase antitumor efficacy, spare normal tissue surround the tumor, or both. However, because changes in tissue density occurring during fractionated particle therapy (e.g., mucus and weight loss) severely affect radiation dose deposition, a main challenge is to verify that the radiation dose is being delivered within the target.

In addition to the previously described possibilities for plan adaptation based on functional imaging, PET technology provides exciting opportunities for particle-beam verification (56). During hadron irradiation, $\beta^{+}$-emitters such as ${ }^{11} \mathrm{C}$ or ${ }^{15} \mathrm{O}$ are produced along the beam path and can be visualized with PET, either online using in-room imaging equipment or offline a few minutes after particle dose administration (57). As a consequence, PET is a noninvasive means for almost instantaneous image-guidance in hadron therapy, with an accuracy of below a few millimeters for estimating the range of proton and carbon ion beams (58). However, biologic washout and patient motion challenge the accuracy of range verification with PET (59). Recently, methods have been developed that allow automatic range assessment and uncertainty estimation already during the treatment planning process for particle therapy (60).

A first small clinical study using an inroom PET system for proton range verification demonstrated its potential for in vivo treatment monitoring in proton therapy (61). A larger clinical study (MIRANDA) is currently recruiting patients in Germany with the aim of investigating the clinical feasibility and effectiveness of PET quality assurance to promote the accuracy of proton and carbon ion therapy (62).

Because of the steep dose gradients obtained with particle-beam therapy, it may be ideal for the clinical realization of biologically adapted therapy based on functional PET or MRI. A few planning studies have investigated the potential of proton-beam therapy for dose painting and found better organ-at-risk sparing and therefore increased flexibility in terms of local dose escalation, for example $(63,64)$. Also, the higher linearenergy transfer of heavy ions may be of great value in overcoming local radiation resistance, such as that induced by tumor hypoxia (65).

Finally, PET may facilitate decision making when photon and proton irradiation are combined (66). On the one hand, particle therapy enables the assignment of highly conformal dose distributions, whereas photon therapy, on the other hand, is robust against motion and other sources of disturbance. As a consequence, depending on the tumor entity and the availability of proton treatment facilities, a combination of protons and photons may be used (66).

\section{MOLECULAR IMAGING IN SYSTEMIC RADIOTHERAPY AND TARGETED THERAPY}

For systemic radiotherapy, that is, administration of radioactive agents that specifically bind to targets on cancer cells, molecular imaging has a special role because drugs can be radiolabeled with therapeutic ( $\alpha$ - or $\beta$-emitting) or diagnostic ( $\gamma$ - or positron-emitting) radionuclides. This concept, called theranostics, is a typical example of personalized treatment, as the targeting of the therapeutic agent can be predicted and followed by a companion diagnostic imaging agent individually in each patient.

Treatment of differentiated thyroid cancer with ${ }^{131} \mathrm{I}$ is the earliest example of theranostics, having been applied for more than $70 \mathrm{y}$. This targeted therapy exploits expression of the sodium/iodine symporter on thyroid cancer cells to detect and treat thyroid cancer with ${ }^{131} \mathrm{I}$, 


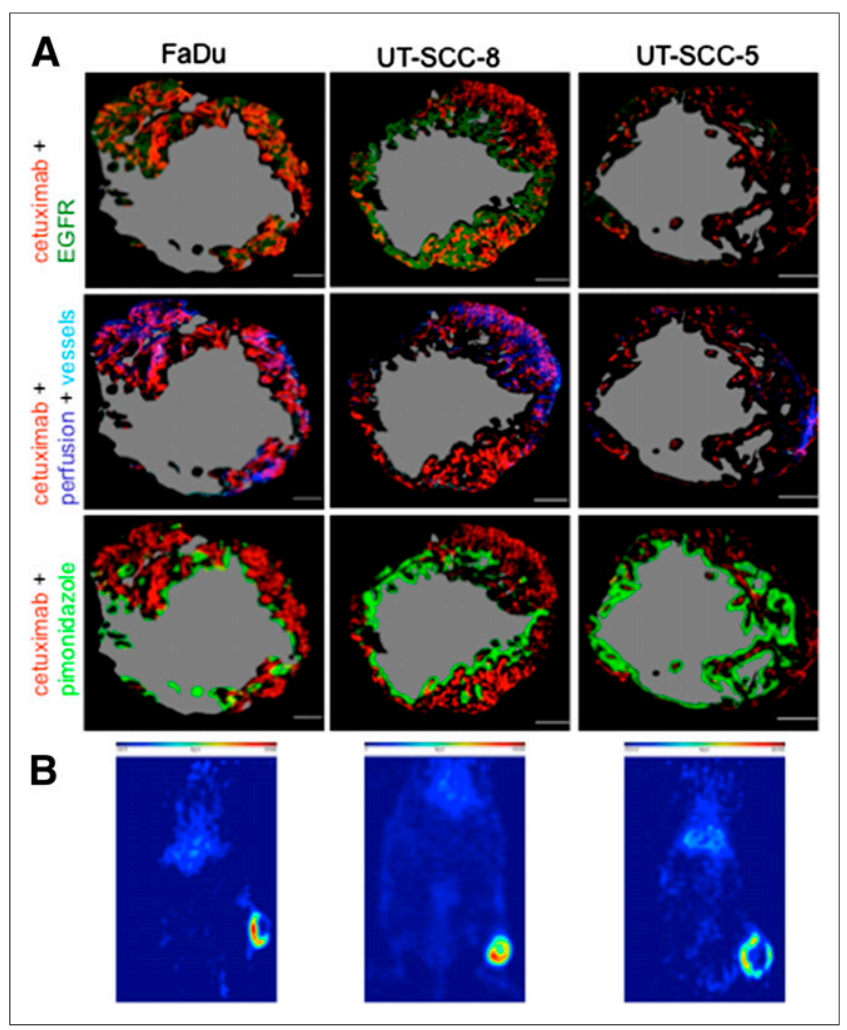

FIGURE 4. (A) Pseudocolored images of representative tumor sections of FaDu, UT-SCC-8, and UT-SCC-5 growing in NMRI (Naval Medical Research Institute) mice. Red = cetuximab; dark green = EGFR; dark blue = perfusion, Hoechst 33342; light blue = vascular endothelium, CD31; light green = hypoxia, pimonidazole; gray = necrotic area. (B) Representative PET images administered ${ }^{86}$ Y-cetuximab. (Reprinted with permission of (78).)

providing an excellent clinical outcome for this disease even when metastatic $(67,68)$. Another example of theranostics is the use of molecular imaging agents to detect and treat metastatic neuroendocrine tumors with radiolabeled somatostatin analogs or radiolabeled metaiodobenzylguanidine (69). With high affinity, these somatostatin analogs specifically target somatostatin subtype receptors and the metaiodobenzylguanidine of noradrenalin transporters, which are overexpressed in neuroendocrine tumors.

More recently, treatment of metastatic prostate cancer with radiopharmaceuticals has been attracting considerable interest $(70,71)$. Patients with bone metastases from castration-resistant prostate cancer amenable to ${ }^{223} \mathrm{Ra}$ treatment are being selected using the $\alpha$-emitting calcium analog ${ }^{223} \mathrm{Ra}$ and bone scintigraphy to depict the sclerotic bone metastases. Although Marie Curie discovered radium isotopes more than a century ago, ${ }^{223} \mathrm{Ra}$ was approved for this indication only in recent years. Novel radiolabeled agents targeting the prostate-specific membrane antigen epitope on prostate cancer cells are being developed for targeting and treating not only bony metastases but also soft-tissue tumors, again directly linking molecular imaging to targeted therapy (72). The monoclonal anti$\mathrm{CD} 20$ antibody conjugated to ${ }^{90} \mathrm{Y},{ }^{90} \mathrm{Y}$-ibritumomab tiuxetan, is a treatment for relapsed or refractory low-grade or follicular nonHodgkin lymphoma that has been approved by the Food and Drug Administration and the European Medicines Agency (73). In the development of this therapeutic approach, the diagnostic companion agent (ibritumomab tiuxetan labeled with the $\gamma$-emitting radionuclide ${ }^{111} \mathrm{In}$ ) was used to evaluate targeting to tumor sites.
This example illustrates the role of molecular imaging in developing targeted therapies. Molecular imaging of radiolabeled drugs not only is applied as an adjunct to radioactive therapeutics but also has been embraced to elucidate mechanisms of action, heterogeneity of target expression, and accessibility of target for drugs that are already widely used in clinical practice, such as trastuzumab in human epithelial growth factor receptor 2-positive metastatic breast cancer $(74,75)$. It is not always necessary to develop new radiopharmaceuticals to monitor and adapt treatment. A typical example is gastrointestinal stromal tumors harboring an activating c-KIT mutation that can be targeted with imatinib. Because imatinib also inhibits hexokinase, the driving enzyme for ${ }^{18} \mathrm{~F}-\mathrm{FDG}$ accumulation in tumor cells, responding gastrointestinal stromal tumors can become completely negative on ${ }^{18} \mathrm{~F}$-FDG PET within days or months before a volume response can be established (76). Conversely, failure to show an ${ }^{18} \mathrm{~F}-\mathrm{FDG}$ response may provide valuable information for changing to surgery or stereotactic radiotherapy for the nonresponding lesions while maintaining the same treatment for the other, still responding, lesions. Additionally, early metabolic response, reflected by decreased ${ }^{18} \mathrm{~F}-\mathrm{FDG}$ uptake early after the start of treatment, may be associated with improved clinical outcome, such as was shown by Demetri et al. (77) in patients with gastrointestinal stromal tumors treated with sunitinib after imatinib failure. This opens a window of opportunity to adapt the dose or change treatment in patients who fail to show a metabolic response.

Apart from their systemic effects, specific radiolabeled agents may well be combined with external-beam radiotherapy. One may postulate the delivery of high doses of systemic radiotherapy to antigen-expressing targets followed by external-beam radiotherapy to the tumor and elective treatment volumes. Consequently, the radiation dose to the target may be increased substantially without harming the surrounding radiation-sensitive normal tissue, thus widening the therapeutic window. Very recently, Koi et al. (78) treated 3 human squamous cell carcinoma models (UT-SCC-5, UT-SCC-8, and FaDu) with the ${ }^{90}$ Y-labeled anti-EGFR antibody cetuximab combined with external-beam radiotherapy (Fig. 4). Varying therapeutic efficacy was reported for the models. As opposed to EGFR expression detected by immunohistochemical staining of tumor sections, ${ }^{86}$ Y-cetuximab PET signal correlated to responsiveness to radiolabeled targeted treatment. At present, 4 clinical studies on the combination of external-beam radiotherapy and targeted agents are registered on www.clinicaltrials.gov covering brain metastases from solid tumors, NSCLC, and diffuse intrinsic pons glioma.

Exploiting molecular imaging to select patients before-and establish nonresponse early during-treatment aids the clinician in earlier adaptation of treatment, sparing the patient the side effects of ineffective drugs and society the cost of futile treatments. However, to wisely use and avoid the unnecessary proliferation of advanced imaging without a proven impact on patient treatment and outcome, it is mandatory to address the development of companion diagnostics during early- and late-phase clinical trials rather than introducing non-evidence-based imaging technology in a clinical setting.

\section{CONCLUSION}

This review article has addressed the various aspects of adapting radiation treatment on the basis of functional imaging. With the ongoing progress in image fusion, treatment plan summation, 
radiation techniques, and new combination treatments (radiotherapy, chemotherapy, monoclonal antibodies, tyrosine kinase inhibitors, and radiolabeled targeted agents), this field has just started to evolve.

\section{REFERENCES}

1. De Ruysscher D, Kirsch CM. PET scans in radiotherapy planning of lung cancer. Radiother Oncol. 2010;96:335-338.

2. Troost EG, Schinagl DA, Bussink J, et al. Innovations in radiotherapy planning of head and neck cancers: role of PET. J Nucl Med. 2010;51:66-76.

3. Grosu AL, Souvatzoglou M, Roper B, et al. Hypoxia imaging with FAZA-PET and theoretical considerations with regard to dose painting for individualization of radiotherapy in patients with head and neck cancer. Int J Radiat Oncol Biol Phys. 2007;69:541-551.

4. Horsman MR, Mortensen LS, Petersen JB, Busk M, Overgaard J. Imaging hypoxia to improve radiotherapy outcome. Nat Rev Clin Oncol. 2012;9:674-687.

5. Thorwarth D, Eschmann SM, Paulsen F, Alber M. A kinetic model for dynamic $\left[{ }^{18} \mathrm{~F}\right]$-Fmiso PET data to analyse tumour hypoxia. Phys Med Biol. 2005;50: 2209-2224.

6. Troost EG, Laverman P, Philippens ME, et al. Correlation of $\left[{ }^{18}\right.$ F]FMISO autoradiography and pimonidazole immunohistochemistry in human head and neck carcinoma xenografts. Eur J Nucl Med Mol Imaging. 2008;35:1803-1811.

7. Zegers CM, van Elmpt W, Wierts R, et al. Hypoxia imaging with $\left[{ }^{18} \mathrm{~F}\right] \mathrm{HX} 4 \mathrm{PET}$ in NSCLC patients: defining optimal imaging parameters. Radiother Oncol. 2013;109:58-64.

8. Buck AK, Herrmann K, Buschenfelde CM, et al. Imaging bone and soft tissue tumors with the proliferation marker $\left[{ }^{18} \mathrm{~F}\right]$ fluorodeoxythymidine. Clin Cancer Res. 2008;14:2970-2977.

9. Idema AJ, Hoffmann AL, Boogaarts HD, et al. $3^{\prime}$-deoxy- $3^{\prime}-{ }^{18}$ F-fluorothymidine PET-derived proliferative volume predicts overall survival in high-grade glioma patients. J Nucl Med. 2012;53:1904-1910.

10. Troost EG, Bussink J, Hoffmann AL, Boerman OC, Oyen WJ, Kaanders JH. ${ }^{18} \mathrm{~F}-$ FLT PET/CT for early response monitoring and dose escalation in oropharyngeal tumors. J Nucl Med. 2010;51:866-874.

11. Aerts HJ, Dubois L, Perk L, et al. Disparity between in vivo EGFR expression and ${ }^{89} \mathrm{Zr}$-labeled cetuximab uptake assessed with PET. J Nucl Med. 2009;50: 123-131.

12. Corcoran EB, Hanson RN. Imaging EGFR and HER2 by PET and SPECT: a review. Med Res Rev. 2014;34:596-643.

13. Laking GR, Price PM. Positron emission tomographic imaging of angiogenesis and vascular function. Br J Radiol. 2014;76(suppl 1).

14. Murakami Y, Takamatsu H, Taki J, et al. ${ }^{18} \mathrm{~F}$-labelled annexin V: a PET tracer for apoptosis imaging. Eur J Nucl Med Mol Imaging. 2004;31:469-474.

15. Pichler BJ, Kolb A, Nagele T, Schlemmer HP. PET/MRI: paving the way for the next generation of clinical multimodality imaging applications. J Nucl Med. 2010;51:333-336.

16. Lagendijk JJ, Raaymakers BW, van Vulpen M. The magnetic resonance imaginglinac system. Semin Radiat Oncol. 2014;24:207-209.

17. Jürgenliemk-Schulz IM, Tersteeg RJ, Roesink JM, et al. MRI-guided treatmentplanning optimisation in intracavitary or combined intracavitary/interstitial PDR brachytherapy using tandem ovoid applicators in locally advanced cervical cancer. Radiother Oncol. 2009;93:322-330.

18. Kharofa J, Morrow N, Kelly T, et al. 3-T MRI-based adaptive brachytherapy for cervix cancer: treatment technique and initial clinical outcomes. Brachytherapy. 2014;13:319-325.

19. Kim S, Loevner L, Quon H, et al. Diffusion-weighted magnetic resonance imaging for predicting and detecting early response to chemoradiation therapy of squamous cell carcinomas of the head and neck. Clin Cancer Res. 2009;15: 986-994.

20. Dirix P, Vandecaveye V, De Keyzer F, Stroobants S, Hermans R, Nuyts S. Dose painting in radiotherapy for head and neck squamous cell carcinoma: value of repeated functional imaging with ${ }^{18} \mathrm{~F}$-FDG PET, ${ }^{18} \mathrm{~F}$-fluoromisonidazole PET, diffusion-weighted MRI, and dynamic contrast-enhanced MRI. J Nucl Med. 2009;50:1020-1027.

21. Schmid MP, Fidarova E, Potter R, et al. Magnetic resonance imaging for assessment of parametrial tumour spread and regression patterns in adaptive cervix cancer radiotherapy. Acta Oncol. 2013;52:1384-1390.

22. Price JM, Robinson SP, Koh DM. Imaging hypoxia in tumours with advanced MRI. Q J Nucl Med Mol Imaging. 2013;57:257-270.

23. Deviers A, Ken S, Filleron T, et al. Evaluation of the lactate-to-N-acetyl-aspartate ratio defined with magnetic resonance spectroscopic imaging before radiation therapy as a new predictive marker of the site of relapse in patients with glioblastoma multiforme. Int J Radiat Oncol Biol Phys. 2014;90:385-393.
24. van der Heide UA, Houweling AC, Groenendaal G, Beets-Tan RG, Lambin P. Functional MRI for radiotherapy dose painting. Magn Reson Imaging. 2012;30: $1216-1223$.

25. Sahani DV, Kalva SP, Hamberg LM, et al. Assessing tumor perfusion and treatment response in rectal cancer with multisection CT: initial observations. Radiology. 2005;234:785-792.

26. Koukourakis MI, Mavanis I, Kouklakis G, et al. Early antivascular effects of bevacizumab anti-VEGF monoclonal antibody on colorectal carcinomas assessed with functional CT imaging. Am J Clin Oncol. 2007;30:315-318.

27. Jiang T, Kambadakone A, Kulkarni NM, Zhu AX, Sahani DV. Monitoring response to antiangiogenic treatment and predicting outcomes in advanced hepatocellular carcinoma using image biomarkers, CT perfusion, tumor density, and tumor size (RECIST). Invest Radiol. 2012;47:11-17.

28. Sauter AW, Winterstein S, Spira D, et al. Multifunctional profiling of non-small cell lung cancer using ${ }^{18} \mathrm{~F}$-FDG PET/CT and volume perfusion CT. J Nucl Med. 2012;53:521-529.

29. Tixier F, Groves AM, Goh V, et al. Correlation of intra-tumor ${ }^{18}$ F-FDG uptake heterogeneity indices with perfusion $\mathrm{CT}$ derived parameters in colorectal cancer. PLoS One. 2014;9:e99567.

30. Jaffray DA. Image-guided radiotherapy: from current concept to future perspectives. Nat Rev Clin Oncol. 2012;9:688-699.

31. van Elmpt W, Ollers M, Dingemans AM, Lambin P, De Ruysscher D. Response assessment using ${ }^{18} \mathrm{~F}$-FDG PET early in the course of radiotherapy correlates with survival in advanced-stage non-small cell lung cancer. J Nucl Med. 2012;53: 1514-1520.

32. Usmanij EA, de Geus-Oei LF, Troost EG, et al. ${ }^{18}$ F-FDG PET early response evaluation of locally advanced non-small cell lung cancer treated with concomitant chemoradiotherapy. $J$ Nucl Med. 2013;54:1528-1534.

33. Bollineni VR, Koole MJ, Pruim J, et al. Dynamics of tumor hypoxia assessed by ${ }^{18} \mathrm{~F}$-FAZA PET/CT in head and neck and lung cancer patients during chemoradiation: possible implications for radiotherapy treatment planning strategies. Radiother Oncol. 2014;113:198-203.

34. Mortensen LS, Johansen J, Kallehauge J, et al. FAZA PET/CT hypoxia imaging in patients with squamous cell carcinoma of the head and neck treated with radiotherapy: results from the DAHANCA 24 trial. Radiother Oncol. 2012;105: $14-20$.

35. Servagi-Vernat S, Differding S, Hanin FX, et al. A prospective clinical study of ${ }^{18} \mathrm{~F}$-FAZA PET-CT hypoxia imaging in head and neck squamous cell carcinoma before and during radiation therapy. Eur J Nucl Med Mol Imaging. 2014;41: 1544-1552.

36. Zips D, Zophel K, Abolmaali N, et al. Exploratory prospective trial of hypoxiaspecific PET imaging during radiochemotherapy in patients with locally advanced head-and-neck cancer. Radiother Oncol. 2012;105:21-28.

37. Hoeben BA, Troost EG, Span PN, et al. ${ }^{18}$ F-FLT PET during radiotherapy or chemoradiotherapy in head and neck squamous cell carcinoma is an early predictor of outcome. J Nucl Med. 2013;54:532-540.

38. Hoeben BA, Troost EG, Bussink J, van Herpen CM, Oyen WJ, Kaanders JH. ${ }^{18} \mathrm{~F}-$ FLT PET changes during radiotherapy combined with cetuximab in head and neck squamous cell carcinoma patients. Nuklearmedizin. 2014;53:60-66.

39. Madani I, Duthoy W, Derie C, et al. Positron emission tomography-guided, focal-dose escalation using intensity-modulated radiotherapy for head and neck cancer. Int J Radiat Oncol Biol Phys. 2007;68:126-135.

40. Leclerc M, Maingon P, Hamoir M, et al. A dose escalation study with intensity modulated radiation therapy (IMRT) in T2N0, T2N1, T3N0 squamous cell carcinomas (SCC) of the oropharynx, larynx and hypopharynx using a simultaneous integrated boost (SIB) approach. Radiother Oncol. 2013;106:333-340.

41. Welz S, Pfannenberg C, Reimold M, et al. Hypoxia dose-escalation with chemoradiation in head and neck cancer: planned interim analysis of a randomized study. Radiother Oncol. 2014;111(suppl):S145.

42. Madani I, Duprez F, Boterberg T, et al. Maximum tolerated dose in a phase I trial on adaptive dose painting by numbers for head and neck cancer. Radiother Oncol. 2011;101:351-355.

43. Heukelom J, Hamming O, Bartelink H, et al. Adaptive and innovative Radiation Treatment FOR improving Cancer treatment outcomE (ARTFORCE): a randomized controlled phase II trial for individualized treatment of head and neck cancer. BMC Cancer. 2013;13:84.

44. Thorwarth D, Mönnich D, Wack LJ, et al. Validation of a hypoxia TCP model and dose painting in HNC: planned interim analysis of a phase II trial. Radiother Oncol. 2014;111(suppl):S123-S124.

45. Leibfarth S, Monnich D, Welz S, et al. A strategy for multimodal deformable image registration to integrate PET/MR into radiotherapy treatment planning. Acta Oncol. 2013;52:1353-1359. 
46. Thorwarth D, Beyer T, Boellaard R, et al. Integration of FDG-PET/CT into external beam radiation therapy planning: technical aspects and recommendations on methodological approaches. Nuklearmedizin. 2012;51:140-153.

47. Olteanu LA, Berwouts D, Madani I, et al. Comparative dosimetry of three-phase adaptive and non-adaptive dose-painting IMRT for head-and-neck cancer. Radiother Oncol. 2014;111:348-353.

48. Houweling AC, Wolf AL, Vogel WV, et al. FDG-PET and diffusion-weighted MRI in head-and-neck cancer patients: implications for dose painting. Radiother Oncol. 2013;106:250-254.

49. Groenendaal G, van den Berg CA, Korporaal JG, et al. Simultaneous MRI diffusion and perfusion imaging for tumor delineation in prostate cancer patients. Radiother Oncol. 2010;95:185-190.

50. Alber M, Thorwarth D. Multi-modality functional image guided dose escalation in the presence of uncertainties. Radiother Oncol. 2014;111:354-359.

51. Aerts HJ, Velazquez ER, Leijenaar RT, et al. Decoding tumour phenotype by noninvasive imaging using a quantitative radiomics approach. Nat Commun. 2014;5:4006.

52. Leijenaar RT, Carvalho S, Velazquez ER, et al. Stability of FDG-PET radiomics features: an integrated analysis of test-retest and inter-observer variability. Acta Oncol. 2013;52:1391-1397.

53. Petit SF, van Elmpt WJ, Oberije CJ, et al. $\left[{ }^{18} \mathrm{~F}\right]$ fluorodeoxyglucose uptake patterns in lung before radiotherapy identify areas more susceptible to radiationinduced lung toxicity in non-small-cell lung cancer patients. Int J Radiat Oncol Biol Phys. 2011;81:698-705.

54. De Ruysscher D, Sharifi H, Defraene G, et al. Quantification of radiation-induced lung damage with CT scans: the possible benefit for radiogenomics. Acta Oncol. 2013;52:1405-1410.

55. Nijkamp J, Rossi M, Lebesque J, et al. Relating acute esophagitis to radiotherapy dose using FDG-PET in concurrent chemo-radiotherapy for locally advanced non-small cell lung cancer. Radiother Oncol. 2013;106:118-123.

56. Parodi K, Paganetti H, Shih HA, et al. Patient study of in vivo verification of beam delivery and range, using positron emission tomography and computed tomography imaging after proton therapy. Int J Radiat Oncol Biol Phys. 2007;68:920-934.

57. Parodi K, Bortfeld T, Haberer T. Comparison between in-beam and offline positron emission tomography imaging of proton and carbon ion therapeutic irradiation at synchrotron- and cyclotron-based facilities. Int J Radiat Oncol Biol Phys. 2008;71:945-956.

58. Seravalli E, Robert C, Bauer J, et al. Monte Carlo calculations of positron emitter yields in proton radiotherapy. Phys Med Biol. 2012;57:1659-1673.

59. Knopf A, Parodi K, Bortfeld T, Shih HA, Paganetti H. Systematic analysis of biological and physical limitations of proton beam range verification with offline PET/CT scans. Phys Med Biol. 2009;54:4477-4495.

60. Frey K, Unholtz D, Bauer J, et al. Automation and uncertainty analysis of a method for in-vivo range verification in particle therapy. Phys Med Biol. 2014; 59:5903-5919.

61. Min $\mathrm{CH}$, Zhu X, Winey BA, et al. Clinical application of in-room positron emission tomography for in vivo treatment monitoring in proton radiation therapy. Int J Radiat Oncol Biol Phys. 2013;86:183-189.
62. Combs SE, Bauer J, Unholtz D, et al. Monitoring of patients treated with particle therapy using positron-emission-tomography (PET): the MIRANDA study. BMC Cancer. 2012;12:133.

63. Flynn RT, Bowen SR, Bentzen SM, Rockwell Mackie T, Jeraj R. Intensitymodulated $\mathrm{x}$-ray (IMXT) versus proton (IMPT) therapy for theragnostic hypoxiabased dose painting. Phys Med Biol. 2008;53:4153-4167.

64. Thorwarth D, Soukup M, Alber M. Dose painting with IMPT, helical tomotherapy and IMXT: a dosimetric comparison. Radiother Oncol. 2008;86:30-34.

65. Bassler N, Jakel O, Sondergaard CS, Petersen JB. Dose- and LET-painting with particle therapy. Acta Oncol. 2010;49:1170-1176.

66. Torres MA, Chang EL, Mahajan A, et al. Optimal treatment planning for skull base chordoma: photons, protons, or a combination of both? Int J Radiat Oncol Biol Phys. 2009;74:1033-1039.

67. Pacini F, Schlumberger M, Harmer C, et al. Post-surgical use of radioiodine $\left({ }^{131} \mathrm{I}\right)$ in patients with papillary and follicular thyroid cancer and the issue of remnant ablation: a consensus report. Eur J Endocrinol. 2005;153:651-659.

68. Sherman SI. Thyroid carcinoma. Lancet. 2003;361:501-511.

69. Wiseman GA, Kvols LK. Therapy of neuroendocrine tumors with radiolabeled MIBG and somatostatin analogues. Semin Nucl Med. 1995;25:272-278.

70. Parker C, Nilsson S, Heinrich D, et al. Alpha emitter radium-223 and survival in metastatic prostate cancer. N Engl J Med. 2013;369:213-223.

71. Sartor O, Coleman R, Nilsson S, et al. Effect of radium-223 dichloride on symptomatic skeletal events in patients with castration-resistant prostate cancer and bone metastases: results from a phase 3, double-blind, randomised trial. Lancet Oncol. 2014;15:738-746.

72. D'Huyvetter M, Xavier C, Caveliers V, Lahoutte T, Muyldermans S, Devoogdt N. Radiolabeled nanobodies as theranostic tools in targeted radionuclide therapy of cancer. Expert Opin Drug Deliv. 2014;11:1939-1954.

73. Scholz CW, Pinto A, Linkesch W, et al. ${ }^{90}$ Yttrium-ibritumomab-tiuxetan as firstline treatment for follicular lymphoma: 30 months of follow-up data from an international multicenter phase II clinical trial. J Clin Oncol. 2013;31:308-313.

74. Mortimer JE, Bading JR, Colcher DM, et al. Functional imaging of human epidermal growth factor receptor 2-positive metastatic breast cancer using ${ }^{64} \mathrm{Cu}$-DOTA-trastuzumab PET. J Nucl Med. 2014;55:23-29.

75. Janjigian YY, Viola-Villegas N, Holland JP, et al. Monitoring afatinib treatment in HER2-positive gastric cancer with ${ }^{18} \mathrm{~F}-\mathrm{FDG}$ and ${ }^{89} \mathrm{Zr}$-trastuzumab PET. J Nucl Med. 2013;54:936-943.

76. Van den Abbeele AD, Gatsonis C, de Vries DJ, et al. ACRIN 6665/RTOG 0132 phase II trial of neoadjuvant imatinib mesylate for operable malignant gastrointestinal stromal tumor: monitoring with ${ }^{18} \mathrm{~F}$-FDG PET and correlation with genotype and GLUT4 expression. J Nucl Med. 2012;53:567-574.

77. Demetri GD, Heinrich MC, Fletcher JA, et al. Molecular target modulation, imaging, and clinical evaluation of gastrointestinal stromal tumor patients treated with sunitinib malate after imatinib failure. Clin Cancer Res. 2009;15:5902-5909.

78. Koi L, Bergmann R, Bruchner K, et al. Radiolabeled anti-EGFR-antibody improves local tumor control after external beam radiotherapy and offers theragnostic potential. Radiother Oncol. 2014;110:362-369. 